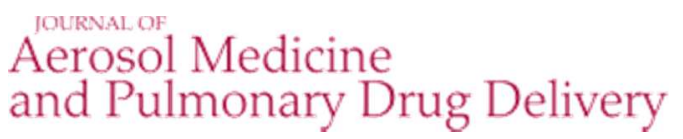

Journal of Aerosol Medicine and Pulmonary Drug Delivery; http://mc.manuscriptcentral.com/aerosol

\title{
Controlled, Parametric, Individualized, 2D and 3D Imaging Measurements of Aerosol Deposition in the Respiratory Tract of Healthy Human Volunteers: In vivo Data Analysis
}

\begin{tabular}{|c|c|}
\hline Journal: & Journal of Aerosol Medicine \\
\hline Manuscript ID: & Draft \\
\hline Manuscript Type: & Original Research \\
\hline Date Submitted by the Author: & $\mathrm{n} / \mathrm{a}$ \\
\hline Complete List of Authors: & $\begin{array}{l}\text { Majoral, Caroline; Air Liquide Santé International - Medical Gases Group, } \\
\text { Centre de Recherche Claude-Delorme } \\
\text { Fleming, John; National Institute of Health Research Biomedical Research } \\
\text { Unit in Respiratory Disease, ; Department of Medical Physics and } \\
\text { Bioengineering, University Hospital Southampton NHS Foundation Trust } \\
\text { Conway, Joy; National Institute of Health Research Biomedical Research } \\
\text { Unit in Respiratory Disease, } \\
\text { Katz, Ira; Air Liquide Santé International - Medical Gases Group, Centre de } \\
\text { Recherche Claude-Delorme } \\
\text { Tossici-Bolt, Livia; Department of Medical Physics and Bioengineering, } \\
\text { University Hospital Southampton NHS Foundation Trust } \\
\text { Pichelin, Marine; Air Liquide Santé International - Medical Gases Group, } \\
\text { Centre de Recherche Claude-Delorme } \\
\text { Montesantos, Spyridon; Air Liquide Santé International - Medical Gases } \\
\text { Group, Centre de Recherche Claude-Delorme } \\
\text { Caillibotte, Georges; Air Liquide Santé International - Medical Gases Group, } \\
\text { Centre de Recherche Claude-Delorme }\end{array}$ \\
\hline Keyword: & Aerosol Distribution, Scintigraphy, inhaled therapy, Clinical Trial \\
\hline
\end{tabular}

\section{SCHOLARONE}

Manuscripts 


\title{
Controlled, Parametric, Individualized, 2D and 3D Imaging Measurements of
} Aerosol Deposition in the Respiratory Tract of Healthy Human Volunteers: In vivo Data Analysis

\author{
RUNNING TITLE: Aerosol Deposition in Healthy Human Volunteers \\ Caroline Majoral*a, John Fleming ${ }^{\mathrm{b}, \mathrm{c}}$, Joy Conway ${ }^{\mathrm{b}, \mathrm{d}}$, Ira Katz ${ }^{\mathrm{a}, \mathrm{e}}$, Livia Tossici-Bolt ${ }^{\mathrm{c}}$, \\ Marine Pichelin ${ }^{a}$, Spyridon Montesantos ${ }^{a}$, Georges Caillibotte ${ }^{a}$
}

\author{
a Medical Gases Group, Air Liquide Santé International, Centre de Recherche Claude- \\ Delorme, Les Loges-en-Josas, France \\ b National Institute of Health Research Biomedical Research Unit in Respiratory Disease, \\ Southampton, UK \\ c Department of Medical Physics and Bioengineering, University Hospital Southampton \\ NHS Foundation Trust, Southampton, UK \\ d Faculty of Health Sciences, University of Southampton, Southampton, UK \\ e Department of Mechanical Engineering, Lafayette College, Easton, PA, USA \\ * Author to whom correspondence should be sent
}

\section{Dr Caroline Majoral}

Air Liquide Santé International, Centre de Recherche Claude-Delorme, 1 chemin de la Porte des Loges - BP 126 Les Loges-en-Josas, 78354 JOUY-EN-JOSAS Cedex FRANCE

Tel: +331390765 27 - Fax: +33139076199

email: caroline.majoral@airliquide.com

Academic degree: PhD 


\author{
Professor John S Fleming \\ Consultant Medical Physicist \\ Southampton Respiratory NIHR Biomedical Research Unit \\ Postal Address: \\ Department of Nuclear Medicine \\ University Hospital Southampton NHS Foundation Trust \\ Mail Pt 26 \\ Tremona Road \\ Southampton \\ SO166YD \\ Tel +44(0)2380796202 \\ E-mail: john.fleming@uhs.nhs.uk \\ Academic degree: PhD
}

Professor Joy H Conway

Professor, Respiratory Sciences and Lung Imaging

Southampton Respiratory Imaging Group

Faculty of Health Sciences

University of Southampton

Level E Centre Block

Room CE115 (mailpoint 886)

Southampton General Hospital

Tremona Road

Southampton 
SO166YD

Tel +44(0)2380796755

E-mail: jhc@soton.ac.uk

Academic degree: PhD

\section{Dr Ira Katz}

Air Liquide Santé International, Centre de Recherche Claude-Delorme, 1 chemin de la Porte des Loges - BP 126 Les Loges-en-Josas, 78354 JOUY-EN-JOSAS Cedex FRANCE

Tel: +33139076511-Fax: +33139076199

email: ira.katz@airliquide.com

Academic degree: PhD

\section{Dr Livia Tossici-Bolt}

Department of Nuclear Medicine

University Hospital Southampton NHS Foundation Trust

Mail Point 26

Tremona Road

Southampton

SO166YD

Tel $+44(0) 2380796628$

E-mail: livia.bolt@uhs.nhs.uk

Academic degree: PhD 


\section{Dr Marine Pichelin}

Air Liquide Santé International, Centre de Recherche Claude-Delorme, 1 chemin de la Porte des Loges - BP 126 Les Loges-en-Josas, 78354 JOUY-EN-JOSAS Cedex FRANCE Tel: +33139076510 - Fax: +33139076199

email: marine.pichelin@airliquide.com

Academic degree: PhD

\section{Dr Spyridon Montesantos}

Air Liquide Santé International, Centre de Recherche Claude-Delorme, 1 chemin de la Porte des Loges - BP 126 Les Loges-en-Josas, 78354 JOUY-EN-JOSAS Cedex FRANCE Tel: +33139076280-Fax: +33139076199

email: spyridon.montesantos@airliquide.com

Academic degree: PhD

\section{Dr Georges Caillibotte}

Air Liquide Santé International, Centre de Recherche Claude-Delorme, 1 chemin de la Porte des Loges - BP 126 Les Loges-en-Josas, 78354 JOUY-EN-JOSAS Cedex FRANCE Tel: +33139076527-Fax: +33139076199

email: georges.caillibotte@airliquide.com

Academic degree: PhD 


\begin{abstract}
(300 words max)
Background

To provide a validation dataset for aerosol deposition modelling, a clinical trial was performed in which the inhalation parameters and the inhaled aerosol were controlled or characterized.
\end{abstract}

\title{
Methods
}

Eleven, healthy, never-smoked, male participants completed the study. Each participant performed two inhalations of Tc-99m labelled aerosol from a vibrating mesh nebulizer, which differed by a single controlled parameter (Aerosol particle size: 'small' or 'large'. Inhalation: 'deep' or 'shallow'. Carrier gas: air or a helium/oxygen mix). The deposition measurements were made by planar imaging, and Single Photon Emission Computed Tomography - Computed Tomography (SPECT-CT).

\section{Results}

The difference between the mean activity measured by imaging and that delivered from the nebulizer was $2.7 \%$, which was not statistically significant.

The total activity deposited was significantly lower in the left lung than in right lung $(p<0.0001)$ with a mean ratio (Left/Right) of $0.87 \pm 0.1$ SD. However, when normalized to lung air volume the left lung deposition was significantly higher $(p=0.0085)$ with a mean ratio of $1.08 \pm 0.12 \mathrm{SD}$. A comparison of the $3 \mathrm{D}$ Central-to-Peripheral $\left(\mathrm{nC} / \mathrm{P}_{3 \mathrm{D}}\right)$ ratio showed that it was significantly higher for left lung $(p<0.0001)$ with a mean ratio (Left/Right) of $1.36 \pm 0.20$ SD.

The effect of particle size was statistically significant on $n C / P_{3 D}$ ratio $(p=0.0014)$, ET deposition $(p=0.0037)$, and $24 h$ clearance $(p<0.0001)$, contrary to the inhalation parameters which showed no effect. 
Two participants inhaled both air and helium-oxygen. The $\mathrm{nC} / \mathrm{P}_{3 \mathrm{D}}$ ratios were lower for the experiments with helium-oxygen than air, suggesting that deposition was more peripheral when breathing helium-oxygen.

\section{Conclusions}

This paper presents the results of an analysis of the in vivo deposition data, obtained in a clinical study designed to provide data for model validation. This study has demonstrated the value of SPECT imaging over planar; the influence of particle size on regional distribution within the lung; and differences in deposition between left and right lungs.

Keywords: aerosol deposition distribution, helium, SPECT, gamma imaging 


\section{Introduction}

To provide a validation dataset for aerosol deposition modeling a clinical study was designed and performed wherein as much as possible the inhalation parameters and the inhaled aerosol were controlled or characterized. A high resolution computed tomography $(\mathrm{HRCT}) \mathrm{x}$-ray scan of the respiratory tract allowed individual morphometric models to be created. Parametric sets of experiments were performed where a single variable was changed, the goal being to isolate model limitations and to guide future developments. The deposition measurements themselves were made by 3-D single photon emission computed tomography (SPECT) imaging in combination) with low resolution (LR) CT that provided anatomical information for attenuation correction. The two LRCT images allowed for co-registration of the SPECT-CT and HRCT images. The spatial deposition data was converted into generational data using the methodology developed by Fleming and his colleagues ${ }^{1}$. Details of the methodology and experimental results are provided in two recent articles ${ }^{2,3}$.

Recently we have published preliminary comparisons of the data with existing models ${ }^{4}$. Other papers analyzing the CT scans have appeared considering morphological characteristics and volume measurements ${ }^{5,6}$. Presented herein are the analyses of the two inhalation experiments for each of the 11 healthy, male volunteers in terms of imaging techniques, dose accountability, 24 hours clearance, comparison of right vs. left lung deposition, and influence of the controlled parameters (particle size, depth of breathing, carrier gas) on aerosol deposition. It is considered that these well controlled and characterized experiments could provide useful information in and of themselves without respect to modeling. 


\section{Materials and Methods}

A brief description of the clinical study and analysis methods appear in this section.

\section{Clinical Study}

The clinical study was performed at the Departments of Nuclear Medicine and Radiology of University Hospital Southampton NHS Foundation Trust Southampton, UK. Approval for the clinical study was obtained from the local research ethics committee and the UK Administration of Radioactive Substances Advisory Committee (ARSAC). Eleven healthy, male participants completed the study, the first five making up a 'pilot' study and the remaining six, the 'main' study. The inclusion criteria were: male volunteers, aged between 18 and 65 years, never-smokers, and lung function tests within the normal range $\left(\mathrm{FEV}_{1}\right.$, FVC, flow volume curves, salbutamol reversibility, TLCO and measurement of lung volumes).

Participants inhaled isotonic saline aerosols seeded with a suspension of Tc-99m labelled particles of human serum albumin (Nanocoll®), GE Healthcare, UK), administered by an AKITA $^{2}{ }^{\circ}$ device (Activaero $\mathrm{GmbH}$, Germany) connected to a vibrating mesh aerosol generator that uses TouchSpray ${ }^{\circledR}$ Technology (Pari Pharma, Germany). The AKITA2 ${ }^{\circledR}$ device allows to control the ventilatory regime and aerosol characteristics. Two distinct but similar devices have been used; one for air and another one for a helium-oxygen mixture as the carrier gas.

After inhalation, 3D-SPECT images were acquired with LRCT scans to account for attenuation. The acquisition was done with a GE Infinia dual head gamma camera with Hawkeye 4 CT attachment (GE Medical Systems, Milwaukee, WI, USA). The initial deposition of aerosol was captured by a $60-\mathrm{sec}$ anterior/posterior planar image of the oropharynx, followed by a further $60-\mathrm{sec}$ anterior/posterior acquisition of the lungs and stomach planar image. Then, a SPECT acquisition was completed, and followed by a 
LRCT image of the thorax at mean tidal breathing, which provided 90 slices with an interslice separation of $4.42 \mathrm{~mm}$. A second planar anterior/posterior image was then acquired. This enabled an estimate of the rate of lung clearance, which could be used to correct activities derived from the SPECT image for this factor. Planar imaging of the nebulizer, the exhalation filter, and the standard was also performed afterwards. The following day a planar anterior/posterior image of the lungs was acquired to calculate the $24 \mathrm{~h}$ clearance, which is a measure of deposition in the conducting airways.

Each subject performed two inhalations at a one week interval, totalling twenty-two 3DSPECT/CT experiments for all participants. For every subject, the two experiments differed by a single controlled parameter: particle size (small or large), depth of breathing (deep or shallow) or carrier gas (air or helium-oxygen mixture). One exception was a pilot experiment with large particles, shallow breathing and air, where the two experiments consisted of inhalation in the erect and then in the supine position. The detailed description of particle size and breathing pattern is given in Table 1 and Table 2 . Detailed descriptions of the study can be found in previous papers ${ }^{2,3}$.

The inspiratory flow rate was controlled by the AKITA $^{\circledR}$ device and set constant to $18 \mathrm{~L} / \mathrm{min}$. However, the time for exhalation and breath hold were not controlled, as breaths were triggered by the subject; thus minute ventilation of $9 \mathrm{~L} / \mathrm{min}$ is only an estimate. Measurements of breath hold and exhalation timing was done with an Embletta sleep diagnostic kit (Emblas, Denver,CO), and are included in the study database ${ }^{2,7}$.

\section{Analysis}

The distribution of activity in the different sections of the airway was determined from analysis of the planar images ${ }^{2}$. Briefly, lung regions of interest (ROI) were obtained from the CT images and the activities in the lungs calculated from the geometric mean counts in the ROIs on anterior and posterior images after correction for radioactive decay, background, mucociliary clearance and attenuation. The activities in the 
trachea/main bronchi, the oropharynx, the stomach, the exhalation filter and the amount remaining in the nebulizer after inhalation were also obtained from planar imaging. Activity in the stomach was added to that in the oropharynx to give an estimated of extra-thoracic deposition. The amount of activity placed into the nebulizer was measured on an isotope calibrator. Dose accountability was performed by calculating the amount of activity leaving the nebulizer and comparing it to that obtained by summing the activity in the body and exhalation filter from the imaging measurements.

The 3D spatial distribution of aerosol deposited in the airways was assessed using several parameters. The process began by dividing the right and left lung regions of interest, determined from the LRCT images, into 10 concentric shells or annuli from centre to periphery, using the hilum as the centre of the transform ${ }^{5}$. The hilum was taken as the first bifurcation of the main bronchus as suggested in the new guidelines for SPECT measurement of aerosol deposition ${ }^{8}$. The shell data were corrected for partial volume $^{9}$ and the $3 \mathrm{D}$ Central-to-Peripheral ratio $\left(C / \mathrm{P}_{3 \mathrm{D}}\right)$ was calculated by dividing the activity in the inner five 3D shells by that in the outer five:

$$
C / P_{3 D}=\frac{\sum_{i=1}^{5} S_{i}}{\sum_{i=6}^{10} S_{i}}
$$

Where $S_{i}$ is the count in shell $i$ on the SPECT image.

As the $C / P$ ratio has been shown to depend on lung shape ${ }^{9}$ the value was normalized to the $3 \mathrm{D} C / \mathrm{P}$ ratio of an air volume image derived from the $\mathrm{LRCT}^{5}$. The $3 \mathrm{D} C / \mathrm{P}$ ratio normalized to lung volume, $\mathrm{nC} / \mathrm{P}_{3 \mathrm{D}}$, is defined by:

$\mathrm{nC} / \mathrm{P}_{3 \mathrm{D}}=\frac{\mathrm{C} / \mathrm{P}_{3 \mathrm{D}} \text { of the initial SPECT image }}{\mathrm{C} / \mathrm{P}_{3 \mathrm{D}} \text { of the air volume image }}$

Other estimations of deposition in the conducting airways were made using the analysis of the SPECT data. Deposition by airway generation was calculated using a conceptual 
model of the spatial arrangement of the lung airway generations within each shell to obtain a mapping to transform shell deposition into generational deposition ${ }^{1}$.

Conducting airways deposition was calculated from the SPECT images in two ways: (i) based on the standard International Commission on Radiological Protection (ICRP) definition of the conducting airways represented by generations 2-15 (CADF, conducting airways deposition fraction) (ii) as defined by Weibel $^{10}$ (wdf), who considered that a fraction of generations 16 to 19 were part of the conducting airways,

$$
w d f=\frac{\sum_{j=2}^{16} G_{j}+0.88 . G_{17}+0.75 . G_{18}+0.5 . G_{19}}{\sum_{j=2}^{23} G_{j}}
$$

The bronchial airways deposition fraction, BADF, defined by generations 2 to 8 was also calculated.

Deposition in the conducting airways was also derived from the $24 \mathrm{~h}$ image. The activity in this image was analyzed as described above and, after all the corrections had been applied, the activity in the each lung was compared to that in the image obtained immediately after inhalation. Clearance was calculated as the percentage loss of activity from the lung at $24 \mathrm{~h}$ relative to the amount in the original image. This is considered to be an estimate of the deposition in the conducting airways ${ }^{11}$.

\section{$\underline{\text { Statistical analysis }}$}

Statistics were achieved using Excel (Microsoft, USA) and SAS v9.2 (SAS Institute Inc., USA). A p value inferior to 0.05 was considered as a significant difference. Random variation between two variables was obtained after linear regression either as the standard error of the estimate or the coefficient of variation. 


\section{Results \\ Analysis of SPECT/CT accuracy \\ Comparison between 3D-SPECT and planar imaging}

The total aerosol deposition in the right and left lungs determined from planar (2D) and SPECT (3D) imaging for all the participants is compared in Figure 1. The planar values were systematically higher than the SPECT by $8.4 \%$. The random difference between these two measurements as determined by the coefficient of variation was $7.6 \%$. These statistics were calculated after correction for mucociliary clearance of the radioaerosol from the lungs. The rate of clearance is determined from the change in count on the anterior images taken before and after the SPECT study, which is assumed to be linear. The mean reduction in counts due to clearance during the SPECT imaging was estimated to be $2.5 \%$ for the right lung and $3.7 \%$ for the left lung. Although small, the clearance should be noted because to do so will reduce differences, as the systematic difference was $-11.9 \%$ before correction, vs. $-8.4 \%$ after correction.

\section{Dose accountability for planar imaging}

Figure 2 shows the total activity leaving the nebulizer assessed from (i) the difference in activity put into the nebulizer and that left after inhalation and (ii) the sum of the activity accounted for in the body and exhalation filter as determined from planar imaging. The mean total activity leaving the nebulizer was $86.5 \pm 29.3 \mathrm{SD} \mathrm{MBq}(n=22)$. The second measurement obtained by summing the activity in the body and exhalation filter gave a mean value of $84.3 \pm 30.9$ SD MBq $(n=22)$. Statistical analysis (Student's t test) gave a $p$ value of 0.0961 , which indicates that there was no significant systematic difference between the two measurements. The random difference as measured by the coefficient of variation was $6.9 \%$. 


\section{4 hours (24h) clearance}

The $24 \mathrm{~h}$ clearance is compared with 3D Central-to-Peripheral ratio normalized to lung volume $\left(n C / P_{3 D}\right)$ and with Outer to Inner ratio $(\mathrm{O} / \mathrm{I})$, as shown on the graph of Figure 3 . The $\mathrm{C} / \mathrm{P}$ ratio has been the common expression of the regional deposition, but the reciprocal of this ratio, the Outer-to-Inner ratio $(\mathrm{O} / \mathrm{I})$, was recommended by the recently published guidelines on the standardisation of techniques for the assessment of aerosol deposition from imaging ${ }^{8}$. Note that though the two methods of presenting the data are essentially equivalent, the resulting statistics in the form of correlation coefficients are different, if just slightly. We will use C/P ratio for the remainder of this paper to be consistent with our previous papers ${ }^{2,3}$.

To compare the spatial distribution of aerosol with the planar $24 \mathrm{~h}$ clearance data in absolute terms, we calculated the following ratio: $\frac{24 h \text { clearance }}{1-24 h \text { clearance }}$ where "24h clearance" represents the conducting airways deposition, and " 1 - $24 \mathrm{~h}$ clearance" the pulmonary deposition. This is an estimate of the central to peripheral deposition ratio based on clearance measurements. This has not been normalized for lung air volume in any way, so it is compared to the non-normalized $c / p$ ratio obtained from SPECT (Figure 4). There is good correlation between the two parameters, but the fraction of deposition in the central conducting airways derived from clearance is much higher than the fraction predicted from spatial analysis of SPECT.

The inherent difficulty of assigning spatial deposition data to anatomical regions has been addressed by Fleming and his colleagues ${ }^{3}$ by overlaying a morphological model in order to assign the deposition to the lung generations. Figure 5 shows the results assuming the standard International Commission on Radiological Protection (ICRP) definition of the conducting airways in the lung represented by generations 2-15 (CADF, conducting airways deposition fraction). 
Figure 6 shows the results obtained by assuming the Weibel definition of the conducting airways. It can be seen that the TB deposition is under represented $(p<0.0001)$ in Figure 5 when using the ICRP definition suggesting that aerosol was being cleared from airways deeper than generation 15 . This is indeed consistent with Weibel's definition of conducting airways that includes a fraction of generations 16 to 19 , thus improving the agreement as shown in Figure 6. The correlation coefficient (95\% confidence interval) between Weibel definition of CADF and $24 \mathrm{~h}$ clearance was $0.60(0.36 ; 0.75)$ (Pearson Correlation Statistics). The coefficient of variation of estimating the $24 \mathrm{~h}$ clearance from the Weibel definition of CADF was $20 \%$. 


\section{Comparison of right vs. left lung aerosol deposition}

Planar imaging techniques used to measure aerosol deposition usually only consider the right lung because the left lung can overlap the stomach where radioactivity deposited in the mouth and swallowed cannot be separated. Thus, it is of interest to compare quantitatively the deposition in right and left lungs as deposition is known to be asymmetric $^{12}$, using the 3D-SPECT images that permit the exclusion of counts in the stomach and more precise attenuation correction. This comparison is provided in terms of total deposition and regional deposition (3D Central-to-Peripheral ratio normalized to lung volume, and bronchial airways deposition fraction, BADF (generations 2-8)). Figure 7 shows left vs. right lungs deposited activity. All the points are below the identity line, indicating that the total activity deposited is significantly lower in the left lung than in right lung $(p<0.0001)$ at a mean ratio (Left/Right) of $0.87 \pm 0.1 S D(n=22)$. However, when normalized to lung air volume using the following ratio:

\section{Activity in Left Lung / Air Volume of Left Lung Activity in Right Lung / Air Volume of Right Lung}

the left lung deposition is significantly higher $(p=0.0085)$ with a mean ratio of $1.08 \pm 0.12 \mathrm{SD}(\mathrm{n}=22)$ (see Figure 8$)$.

A comparison of the 3D normalized Central-to-Peripheral ratio for the left and right lungs showed that it was significantly higher for left lung $(p<0.0001)$ at a mean ratio (Left/Right) of $1.36 \pm 0.20 \mathrm{SD}(\mathrm{n}=22)$ (see Figure 9$)$. These results were confirmed by the calculation of the bronchial airways deposition fraction (BADF), which was also significantly higher for left lung $(p=0.0004)$ than for right lung (Figure 10). The mean ratio of BADF, defined by deposition in generations 2 to 8 , (Left/Right) was $1.68 \pm 0.74$ SD $(n=22)$. 
A comparison of $24 \mathrm{~h}$ clearance for the Right and Left Lungs is shown on Figure11; the difference was significant $(p=0008)$, which gives an independent confirmation of the difference in regional distribution found from $\mathrm{nC} / \mathrm{P}_{3 \mathrm{D}}$ analysis. 
Influence of the controlled parameters (particle size, depth of breathing, carrier gas) on aerosol deposition

Influence of particle size and depth of breathing

Figure 12 reproduces the graph in Figure 6 of the $24 \mathrm{~h}$ clearance vs. conducting airways deposition fraction calculated with the Weibel Model, but with colors and shapes of the symbols to differentiate the individual inhalation experiments. There is a clear distinction that large particles (in red) are more centrally deposited than small particles (blue), but no clear distinction between the deep (squares) and shallow (triangles) breathing patterns.

These trends are confirmed by statistical analysis. $\mathrm{nC} / \mathrm{P}_{3 \mathrm{D}}$, extra-thoracic $(\mathrm{ET})$ deposition (from planar images), $24 \mathrm{~h}$ clearance (from planar images), and total airway deposition in the trachea and right and left lungs (from planar images), were compared for the four following combinations: (i) large particles and shallow inhalation, (ii) large particles and deep inhalation, (iii) small particles and shallow inhalation, and (iv) small particles and deep inhalation. 3D normalized $\mathrm{nC} / \mathrm{P}_{3 \mathrm{D}}$ ratio, $\mathrm{ET}$ deposition, and $24 \mathrm{~h}$ clearance were almost all higher for large particles whatever the breathing regime, whereas total airway deposition was higher for small particles. Two-factor Analysis Of Variance showed that the effect of particle size was statistically significant on $n C / P_{3 D}$ ratio $(p=0.0014)$, ET deposition $(p=0.0037), 24 h$ clearance $(p<0.0001)$ and total airway deposition $(p=0.0366)$, whereas the effect of inhalation parameters was not statistically significant on $n C / P_{3 D}$ ratio $(p=0.3949)$, ET deposition $(p=0.9558)$, 24 h clearance $(p=0.7097)$, or total airway deposition $(p=0.3481)$.

Figure 13 shows total airway deposition, ET deposition, 24h clearance (conducting airways deposition) and 1-24h clearance (pulmonary deposition) for large and small particles, and deep and shallow inhalation; illustrating that the differences in deposition between large and small particles, were more significant than those occurring due to changes from deep to shallow inhalation regimes. 
Influence of the carrier gas: aerosol deposition breathing helium-oxygen vs. Air

In Figure 12 the red clear symbols representing experiments with large particles and helium-oxygen are all below the solid red ones (representing experiments with large particles and air), meaning that breathing helium-oxygen has reduced deposition in the conducting airways determined from the planar $24 \mathrm{~h}$ clearance measurement. The effect of carrier gas can be further studied using the generational representation of deposition based on the 3D spatial images and the conceptual morphological model $)^{1}$ as shown in Figure 14. The results are expressed as a percentage of deposited aerosol in the right and left lungs separately, from generations 2 (lobar bronchi) to 23 (alveoli) for participants H05A (Air) vs. H05B (helium), and H06A (Air) vs. H06B (helium). It is noted that right vs. left differences are less for helium-oxygen than for air, though the number of participants is insufficient to know if this is a consistent effect. However, this observation suggests that the heterogeneity of aerosol deposition with helium-oxygen as the carrier

$\mathrm{nC} / \mathrm{P}_{3 \mathrm{D}}$ was 1.95 and 2.94 for the right and left lungs, respectively for H05A (Air), vs. 1.38 and 1.69 for H05B (helium-oxygen). For $\mathrm{H06A}$ (Air), nC/ $\mathrm{P}_{3 \mathrm{D}}$ was 1.48 and 2.40 for right and left lungs respectively, vs. 1.23 and 1.47 for H06B (helium-oxygen). The 3D Central-to-Peripheral ratios normalized to lung volume were lower for the experiments with helium-oxygen than air, for the two participants and both for right and left lungs. These results suggest that deposition is less central and more peripheral when breathing helium-oxygen.

Comparison of deposition when breathing air vs. helium-oxygen for all the participants inhaling large particles is given in Table 3, in the following regional terms: extra-thoracic (ET) deposition, 3D Central-to-Peripheral ratios normalized to lung volume (mean of Right+Left lungs), 24h clearance (mean of Right+Left lungs), total activity deposited in 
the lungs and BADF. For the "air + large particles" combination, the number of tests was $10(n=10)$, and for "helium-oxygen + large particles" combination, the number was $3(n=3)$. These data support the premise that breathing helium-oxygen can increase peripheral deposition compared to central deposition. 


\section{Discussion}

The objective of this paper was to present the results of an analysis of the in vivo deposition data obtained in a clinical study, designed to provide data for model validation, but that also allows for the study of the influence of several inhalation parameters on aerosol deposition. The focus has been on comparisons of results from the $2 \mathrm{D}$ and $3 \mathrm{D}$ imaging approaches, thus maintaining continuity with the previous imaging literature; but also providing a bridge to future analysis that will focus on the inherent 3D character of the data and direct correlation with anatomy obtained from the CT images.

The analysis described in this paper does not conform exactly to the new standard guidelines for using planar imaging ${ }^{13}$ and $\mathrm{SPECT}^{8}$ for measuring aerosol deposition. This was done to keep consistency with the companion paper describing the acquisition of the image database on these healthy participants. The deviations from the guidelines are in the planar analysis: (i) the use of a $10 \%$ contour to determine the lung region of interest (ROI) rather than the $15 \%$ recommended, (ii) the calculation of $\mathrm{C} / \mathrm{P}$ (central to peripheral) ratios rather than $\mathrm{O} / \mathrm{I}$ (Outer to inner) ratios. It is felt that the difference in the planar ROI analysis technique is unlikely to make any significant difference to the results. The effect of calculating $\mathrm{O} / \mathrm{I}$ ratios instead of $\mathrm{C} / \mathrm{P}$ has been investigated to some extent in this paper (Figure 3) and has been shown as expected to make only a very minor difference to the analysis.

The analysis began with SPECT/CT accuracy. The comparison between total aerosol deposition in the lungs determined from planar (2D) and SPECT (3D) imaging showed that planar imaging gave slightly higher deposited activity compared to the SPECT, but that taking the clearance into account enabled these differences to be reduced.

The dose accountability for planar imaging showed good correlation between the total activity leaving the nebulizer assessed from (i) the difference in activity put into the nebulizer and that left after inhalation and (ii) the sum of the activity accounted for in 
the body and exhalation filter. The difference between the mean activities was $2.7 \%$, well within the $10 \%$ limit set by the ISAM guidelines ${ }^{13}$.

The study enabled measures of regional distribution of aerosol deposition from the initial imaging measurement to be compared to the $24 \mathrm{~h}$ clearance measurements, which are thought to be a good measure of conducting airways deposition in healthy volunteers. The division of the lung into inner and outer spatial zones for analysis of the initial deposition pattern is known to only loosely approximate to conducting (central) and alveolated (peripheral airways). This is particularly true for 2D imaging, but even in 3D there is a significant contribution of alveloated airways in the inner central region, and the conducting airways also penetrate out almost to the edge of the lung. The observation that the fraction in the inner central region is lower than the clearance observed on the $24 \mathrm{~h}$ measurements suggests that the influence of conducting airways in the outer peripheral region outweighs the impact of peripheral airways in the inner central region (Figure 4). This seems consistent with the suggestion by Weibel ${ }^{10}$ that the conducting airways are found as deep into the airway tree as generation 19 .

The importance of deposition in the smaller conducting airways is confirmed in the analysis of deposition by generation. The results obtained assuming the standard International Commission on Radiological Protection (ICRP) definition of the conducting airways in the lung represented by generations $2-15^{14}$ and those obtained by assuming the Weibel definition of the conducting airways, suggested that aerosol was being cleared from airways deeper than generation $15^{10}$. The majority of these airways are located in shells 9 and 10 close to the other edge of the lung.

The acquisition of 3D data from SPECT enabled detailed study on deposition in the left lung without significant impact of activity in the stomach ${ }^{15}$. Comparison of right vs. left lung aerosol deposition showed that the total activity deposited was significantly lower in the left lung than in right lung. This was expected due to the lower air volume in the left lung. However the L/R ratio of deposition was in fact a little higher than the ratio of air 
volumes, which was $0.83^{5}$. When normalized to lung air volume the left lung deposition was significantly higher than the right, demonstrating that left lung deposition was greater than would be expected relative to the right on the basis of lung air volume alone.

It was also noted that central deposition was significantly higher for left lung both from analysis of the initial deposition pattern and the $24 \mathrm{~h}$ clearance. The difference between right and left lungs in terms of $24 \mathrm{~h}$ clearance was considerably smaller than the difference for $\mathrm{nC} / \mathrm{P} 3 \mathrm{D}$ or BADF (generations 2 to 8 ). BADF, and to a lesser extent $\mathrm{nC} / \mathrm{P} 3 \mathrm{D}$, depend on deposition in the bronchial airways up to generation 8 . This suggests that most of the difference in the left and right lung deposition occurs in the first few generations rather than the smaller conducting airways. The $24 \mathrm{~h}$ measurement will be dominated by the deposition in the smaller conducting airways which have a much larger relative volume. The finding that the left lung has greater central deposition than the right is consistent with previous results. Studies aiming to target aerosol delivery using bolus techniques have shown that shallow boluses targeted toward the central conducting airways are preferentially deposited in the left lung, whereas those targeted toward the lung periphery are more evenly distributed between left and right lungs ${ }^{12}$.

The study on the influence of particle size and depth of breathing showed, as expected, a significant effect on deposition pattern due to particle size. The smaller particles had a slightly increased whole lung deposition, $87 \%$ compared to $77 \%$. These values were considerably higher than those predicted from conventional empirical models of aerosol deposition in the lung. These generally predict that for particles of this size, more of the aerosol will be exhaled ${ }^{14,15}$. This may be related to hygroscopic growth within the lung causing particles that would have been exhaled to be deposited in the lung due to their increased size. Future work will involve the application of computer models of deposition to this data in order to provide an explanation for this observation.

The larger particles had greater deposition in the ET region and in the central pulmonary airways. This was demonstrated in both the initial deposition pattern (nC/P3D) and in 
the $24 \mathrm{~h}$ clearance results and is consistent with expected results from modeling ${ }^{14}$ and from previous experimental studies ${ }^{16}$. The influence of inhalation parameters was less clear. Deep inhalation resulted in higher total deposition in the lung with less in the ET region and central pulmonary airways. However this effect was smaller and more variable and not statistically significant. The influence of using helium-oxygen as the carrier gas compared to air has been studied for the larger particles. Breathing heliumoxygen increased total lung deposition and reduced deposition in the ET region and the conducting airways fraction of pulmonary deposition ${ }^{17,18}$. However, the number of studies performed was considered too small to perform statistical analysis. Investigation of the estimated deposition per generation suggested that helium-oxygen may also decrease the heterogeneity of aerosol deposition between left and right lungs. These results suggest that altering the carrier gas may help in targeting aerosol to specific parts of the airway tree. The reason for this effect is currently being studied using computer modeling of deposition. There may be effects due to the different density and viscosity of helium-oxygen.

The $24 \mathrm{~h}$ clearance measurement was particularly good at distinguishing the effect of particle size, providing complete separation between the two groups of measurements using the large and small particle sizes. By contrast, the conducting airways deposition fraction using the Weibel definition of airways (wdf) was less good at separating the groups. Although there was significant correlation between wdf and $24 \mathrm{~h}$ clearance, there was considerable overlap in wdf values between the groups. This points to a limitation in precision in the shell to generation conversion. This can be explained by the significant proportion of conducting airway deposition found in the small airways of generations 1519 mentioned above. The conceptual model used to analyze the images treats the acinus as a unit consisting of airways from generation 15-23. It therefore cannot easily separate deposition on these peripheral conducting airways and the alveoli, giving a limit on its ability to assess total conducting airways deposition. 
In conclusion this study has demonstrated the value of three dimensional radionuclide imaging in assessing the regional distribution of aerosol deposition within the lung. The expected influence of particle size has been demonstrated and new data on the regional distribution pattern has been presented using comparison of initial deposition with $24 \mathrm{~h}$ clearance measurements. New information on the difference in deposition in left and right lungs has also been provided.

\section{Acknowledgments}

The authors thank Benoît Piednoit for his help on the statistical analysis. John Fleming and Joy Conway acknowledge the support of the Southampton Respiratory Biomedical Research Unit funded by the U.K. National Institute for Health Research.

\section{Author Disclosure Statement}

No conflicts of interest exist. 


\section{References}

1. Fleming JS, Sauret V, Conway JH, and Martonen TB: Validation of the conceptual anatomical model of the lung airway. J.Aerosol Med. 2004;17:260-269.

2. Conway JH, Fleming JS, Majoral C, Katz I, Perchet D, Peebles C, TossiciBolt L, Collier L, Caillibotte G, Pichelin M, Sauret-Jackson V, Martonen T, Apiou-Sbirlea G, Muellinger B, Kroneberg P, Gleske J, Scheuch G, Texereau J, Martin A, Montesantos S, and Bennett M: Controlled, Parametric, Individualized, 2-D and 3-D Imaging Measurements of Aerosol Deposition in the Respiratory Tract of Healthy Human Subjects for Model Validation. J.Aerosol Sci. 2012;52:1-17.

3. Fleming J, Conway J, Majoral C, Tossici-Bolt L, Katz I, Caillibotte G, Perchet D, Pichelin M, Muellinger B, Martonen T, Kroneberg P, and piou-Sbirlea G: The use of combined single photon emission computed tomography and $X-$ ray computed tomography to assess the fate of inhaled aerosol. J Aerosol Med.Pulm.Drug Deliv. 2011;24:49-60.

4. Katz I, Pichelin M, Caillibotte G, Montesantos S, Majoral C, Martonen T, Fleming J, Bennett $M$, and Conway J: Controlled, Parametric, Individualized, 2-D and 3-D Imaging Measurements of Aerosol Deposition in the Respiratory Tract of Healthy Human Subjects: Preliminary Comparisons with Simulations. Aerosol Sci.Tech. 2013; 47:714-723.

5. Fleming J, Conway J, Majoral C, Bennett M, Caillibotte G, Montesantos S, and Katz I: A technique for determination of lung outline and regional lung air volume distribution from computed tomography. J Aerosol Med Pulm.Drug Deliv. 2013;(In press). 
6. Montesantos S, Katz I, Majoral C, Pichelin M, Dubau C, Piednoir B, Conway J, Texereau J, and Caillibotte G: Airway morphology from HRCT in healthy subjects and patients with moderate persistent asthma. Anatomical Record. 2013;(In press).

7. Conway J, Fleming J, Majoral C, Katz I, Perchet D, Peebles C, Tossici-Bolt L, Collier L, Caillibotte G, Pichelin M, Sauret-Jackson V, Martonen T, ApiouSbirlea A, Muellinger B, Kroneberg P, Gleske J, Scheuch G, Texereau J, Martin A, Montesantos S, and Bennett M: Controlled, Parametric, Individualized, 2-D and 3-D Imaging Measurements of Aerosol Deposition in the Respiratory Tract of Healthy Human Subjects for Model Validation. J.Aerosol Sci. 2012;52:1-17.

8. Fleming J, Bailey D, Chan H-K, Conway J, KuehI P, Laube B, and Newman S: Standardization of techniques for using single photon emission computed tomography (SPECT) for aerosol deposition assessment of orally inhaled products. J Aerosol Med Pulm.Drug Deliv. 2012;25 Suppl 1:S29-51.

9. Fleming J.S., Sauret V, Conway J.H., Holgate ST, Bailey AG, and Martonen T.B.: Evaluation of the accuracy and precision of lung aerosol deposition measurements from SPECT using simulation. J.Aerosol Med. 2000;13:187198.

10. Weibel E (1963) Morphometry of the human lung. Berlin, Heidelberg Springer Verlag

11. Ilowite J, Smaldone G, Perry R, Bennett W, and Foster W: Relationship between tracheobronchial particle clearance rates and sites of initial deposition in man. Arch.Environ.Health. 1989;44:267-273. 
12. Moller W, Meyer G, Scheuch G, Kreyling WG, and Bennett WD: Left-to-right asymmetry of aerosol deposition after shallow bolus inhalation depends on lung ventilation. J Aerosol Med.Pulm.Drug Deliv. 2009;22:333-339.

13. Newman S, Bennett W, Biddiscombe M, Devadason S, Dolovich M, Fleming J, Haeussermann S, Kietzig C, Kuehl P, Laube B, Sommerer K, Taylor G, Usmani O, and Zeman K: Standardization of techniques for using planar (2D) imaging for aerosol deposition assessment of orally inhaled products. J.Aerosol Med.Pulm.Drug Deliv. 2012; 25(S1):S10-S28.

14. ICRP (1994) Human respiratory tract model for radiological protection. Vol. 24. ICRP Publication 66 ed. Pergamon

15. Fleming JS, Epps BP, Conway JH, and Martonen TB: Comparison of SPECT aerosol deposition data with a human respiratory tract model. J.Aerosol Med. 2006;19:268-278.

16. Usmani OS, Biddiscombe MF, and Barnes PJ: Regional lung deposition and bronchodilator response as a function of beta2-agonsit particle size. Am.J.Respir.Crit.Care Med. 2005;172:1497-1504.

17. Corcoran TE, Gamard S: Development of aerosol drug delivery with helium oxygen gas mixtures. J.Aerosol Med. 2004;17:299-309.

18. Peterson JB, Prisk GK, and Darquenne C: Aerosol deposition in the human lung periphery is increased by reduced-density gas breathing. J.Aerosol Med.Pulm.Drug Deliv. 2008;21:159-168. 
Individual to whom reprint requests should be directed:

Caroline Majoral

Address: Air Liquide Santé International, Centre de Recherche Claude-Delorme, 1 chemin de la Porte des Loges - BP 126 Les Loges-en-Josas, 78354 JOUY-EN-JOSAS

Cedex FRANCE 


\section{Tables}

\begin{tabular}{|l|l|}
\hline \multicolumn{2}{|l|}{ Aerosol size } \\
\hline \multirow{2}{*}{ Large } & MMAD $=5.8 \mu \mathrm{m}$ \\
\cline { 2 - 2 } & GSD $=1.6$ \\
\hline \hline \multirow{2}{*}{ Small } & MMAD $=3.1 \mu \mathrm{m}$ \\
\cline { 2 - 2 } & GSD $=1.5$ \\
\hline
\end{tabular}

Table 1 Aerosol description. MMAD (Mass Median Aerodynamic Diameter), GSD (Geometric Standard Deviation) 
Table 2 Breathing pattern description. TV (Tidal volume), f (Breathing frequency in breaths per minute), Ti (Inspiration time), VE (Minute ventilation defined by $\mathrm{VE}=\mathrm{TV} \times \mathrm{f}$ ), IFR (Inspiratory flow rate defined by $\mathrm{IFR}=\frac{\mathrm{TV}}{\mathrm{Ti}}$ ) 


\begin{tabular}{|l|l|l|}
\hline & $\begin{array}{l}\text { air }+ \text { large particles } \\
(\mathrm{n}=10) \\
(\text { mean } \pm 95 \% \text { confidence } \\
\text { interval) }\end{array}$ & $\begin{array}{l}\text { helium-oxygen + large } \\
\text { particles }(\mathrm{n}=3) \\
\text { (mean } \pm 95 \% \text { confidence } \\
\text { interval) }\end{array}$ \\
\hline $\begin{array}{l}\text { ET deposition (percentage of } \\
\text { inhaled) }\end{array}$ & $19.2 \pm 4.7 \%$ & $10.4 \pm 5.0 \%$ \\
\hline $\begin{array}{l}\text { nC/P 3D (mean of Right+Left } \\
\text { lungs) }\end{array}$ & $2.42 \pm 0.45$ & $1.56 \pm 0.26$ \\
\hline $\begin{array}{l}\text { 24h clearance (mean of } \\
\text { Right+Left lungs) }\end{array}$ & $0.51 \pm 0.04$ & $0.41 \pm 0.04$ \\
\hline total airway deposition \\
(percentage of inhaled)
\end{tabular}

Table 3 Extra-thoracic deposition, central-to-peripheral ratio, 24h clearance, total airway deposition and BADF for "air+large particles" vs. "heliumoxygen+large particles" 


\section{List of Figures}

Figure 1 Total deposition in each lung determined from planar (2D) and SPECT (3D) imaging, corrected for mucociliary clearance. The line is the identity line.

- Figure 2 The total activity leaving the nebulizer assessed from (i) the difference in activity put into the nebulizer and that left after inhalation and (ii) the sum of the activity accounted for in the body and exhalation filter by planar imaging, corrected for mucociliary clearance. The line is the identity line.

- Figure 3 The variation of $24 \mathrm{~h}$ clearance with 3D normalized central-to-peripheral ratio and Outer to Inner ratio. The lines are the least squares linear regressions.

- Figure 4 The variation of "24h clearance / (1 - 24h clearance)" with 3D central-toperipheral ratio. The dashed line is the identity line; the solid line is the least squares linear regression.

- Figure 5 The variation of $24 \mathrm{~h}$ clearance with the estimated fractional deposition in generations $2-15$ (CADF). The line is the identity line.

- Figure 6 The variation of $24 \mathrm{~h}$ clearance with conducting airways deposition fraction assuming the definition of the conducting airways in the Weibel Model. The line is the identity line.

- Figure 7 Comparison of deposition in the right and left lungs from 3D-SPECT. The line is the identity line.

- Figure 8 Deposition of activity in right and left lungs from 3D-SPECT normalized to lung volume. The line is the identity line.

- Figure 9 Spatial deposition: 3D normalized Central-to-Peripheral ratio in right and left lungs. The line is the identity line.

- Figure 10 Airway deposition: bronchial airways deposition fraction (BADF, generations 2-8) in right and left lungs. The line is the identity line.

- Figure $1124 \mathrm{~h}$ clearance in right and left lungs. The line is the identity line.

- Figure 12 The variation of $24 \mathrm{~h}$ clearance with conducting airways deposition fraction assuming the definition of the conducting airways in the Weibel Model. The 
parameters have been differentiated with symbols and color codes. The line is the identity line.

- Figure 13 Total airway deposition, ET deposition, and 24h clearance (conducting airways deposition) for large and small particles, and deep and shallow regimes. Histograms are the Means, error bars the Standard Deviation.

- Figure 14 Generational deposition for healthy participants whose varying parameter was carrier gas. 


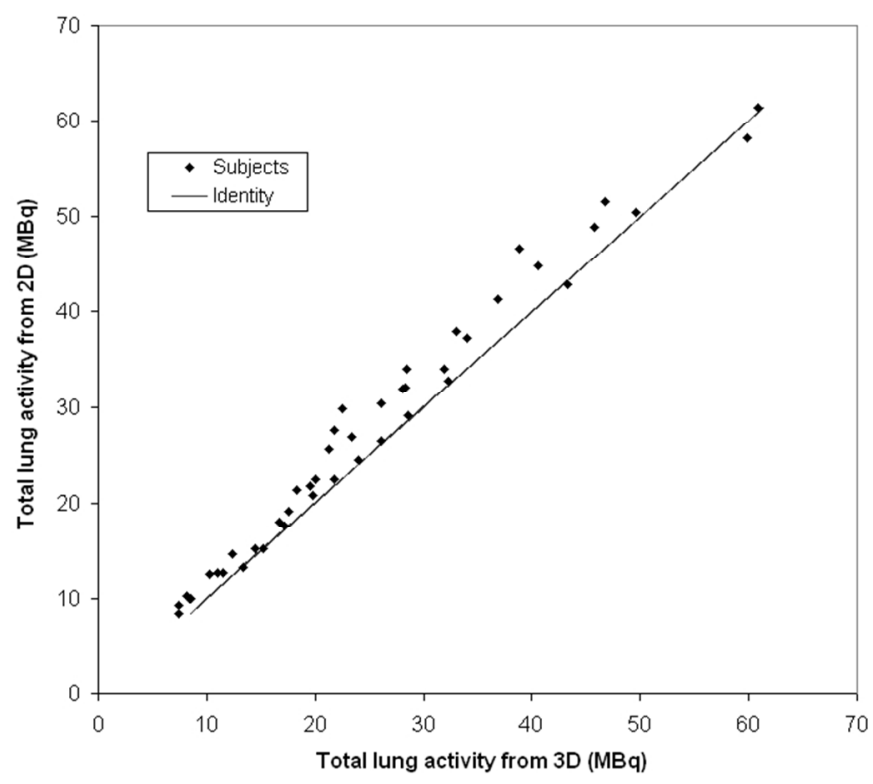

Figure 1 Total deposition in each lung determined from planar (2D) and SPECT (3D) imaging, corrected for mucociliary clearance. The line is the identity line. $256 \times 158 \mathrm{~mm}(96 \times 96 \mathrm{DPI})$ 


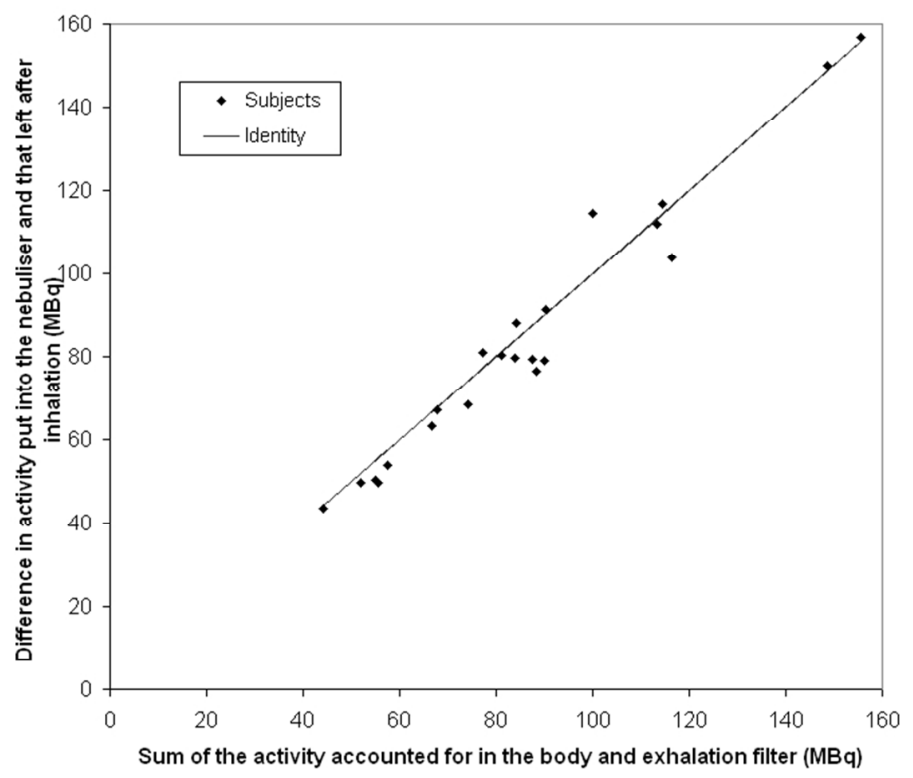

Figure 2 The total activity leaving the nebulizer assessed from (i) the difference in activity put into the nebulizer and that left after inhalation and (ii) the sum of the activity accounted for in the body and exhalation filter by planar imaging, corrected for mucociliary clearance. The line is the identity line. $256 \times 158 \mathrm{~mm}(96 \times 96 \mathrm{DPI})$ 


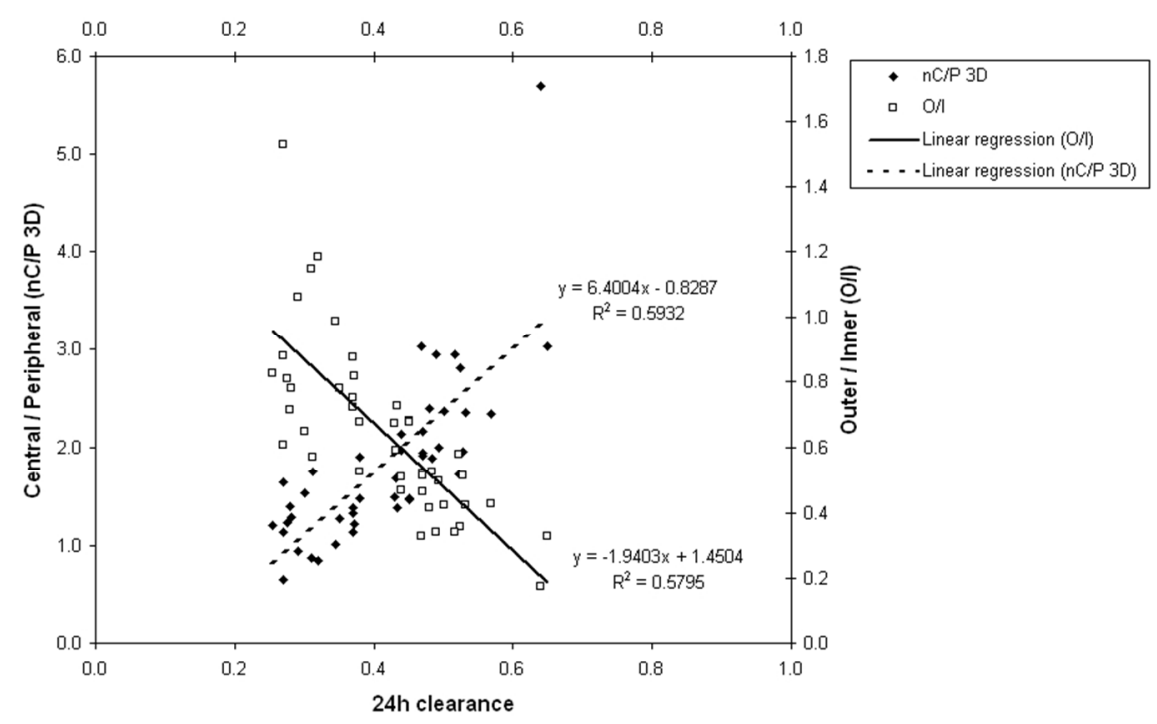

Figure 3 The variation of $24 \mathrm{~h}$ clearance with 3D normalized central-to-peripheral ratio and Outer to Inner ratio. The lines are the least squares linear regressions.

$256 \times 158 \mathrm{~mm}(96 \times 96 \mathrm{DPI})$ 


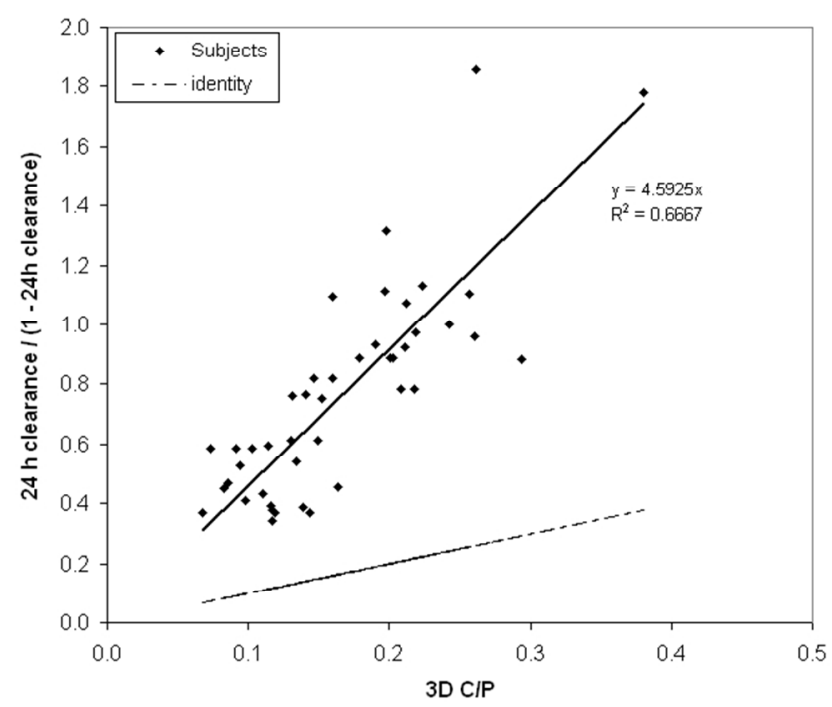

Figure 4 The variation of " $24 \mathrm{~h}$ clearance / ( $1-24 \mathrm{~h}$ clearance)" with 3D central-to-peripheral ratio. The dashed line is the identity line; the solid line is the least squares linear regression. $256 \times 158 \mathrm{~mm}$ (96 x 96 DPI) 


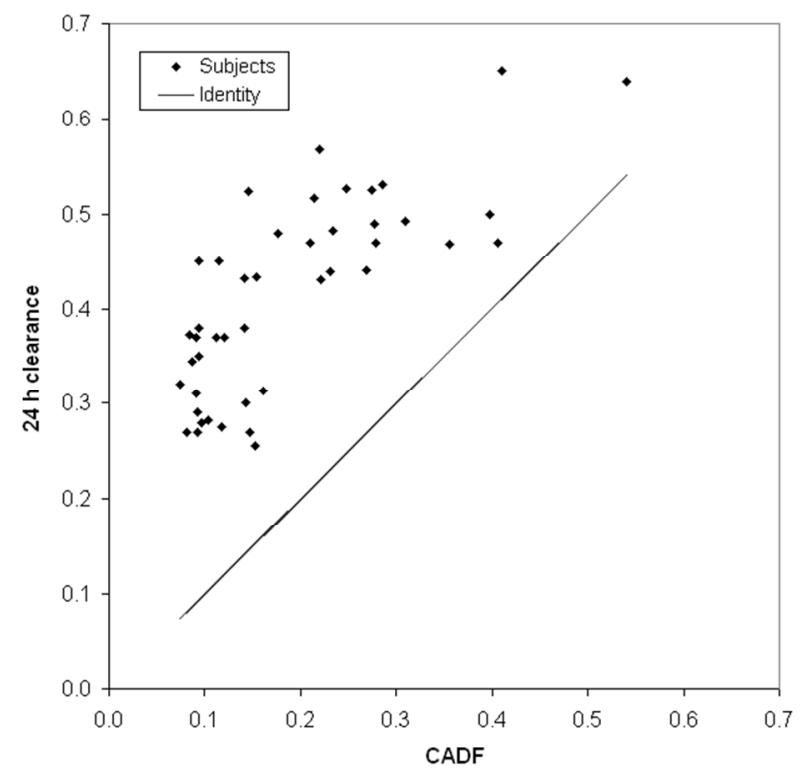

Figure 5 The variation of $24 \mathrm{~h}$ clearance with the estimated fractional deposition in generations 2-15 (CADF). The line is the identity line.

$256 \times 158 \mathrm{~mm}(96 \times 96 \mathrm{DPI})$ 
Figure 6 The variation of 24h clearance with conducting airways deposition fraction assuming the definition of the conducting airways in the Weibel Model. The line is the identity line. $256 \times 158 \mathrm{~mm}(96 \times 96 \mathrm{DPI})$ 
8

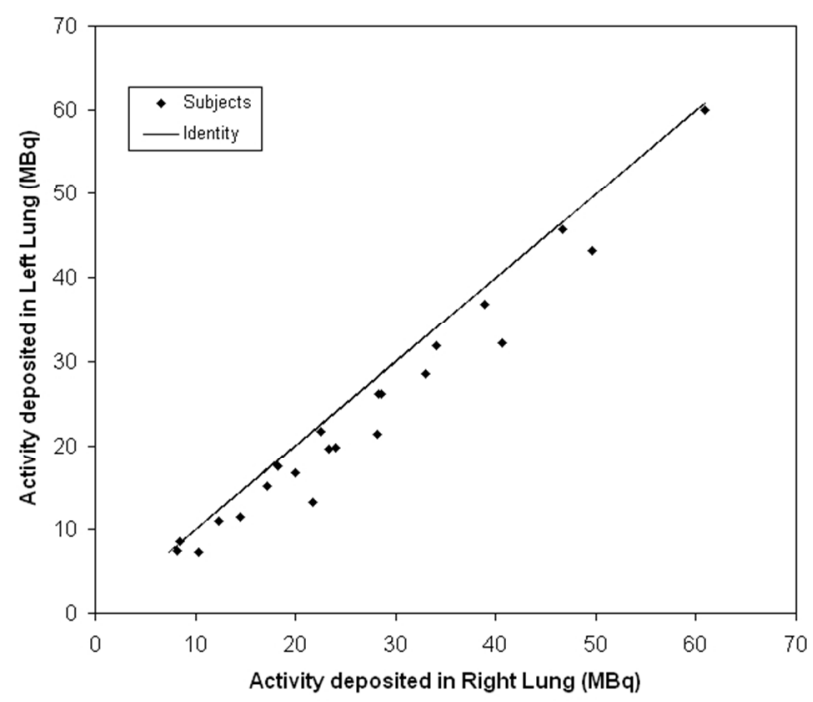

Figure 7 Comparison of deposition in the right and left lungs from 3D-SPECT. The line is the identity line. $256 \times 159 \mathrm{~mm}(96 \times 96 \mathrm{DPI})$ 


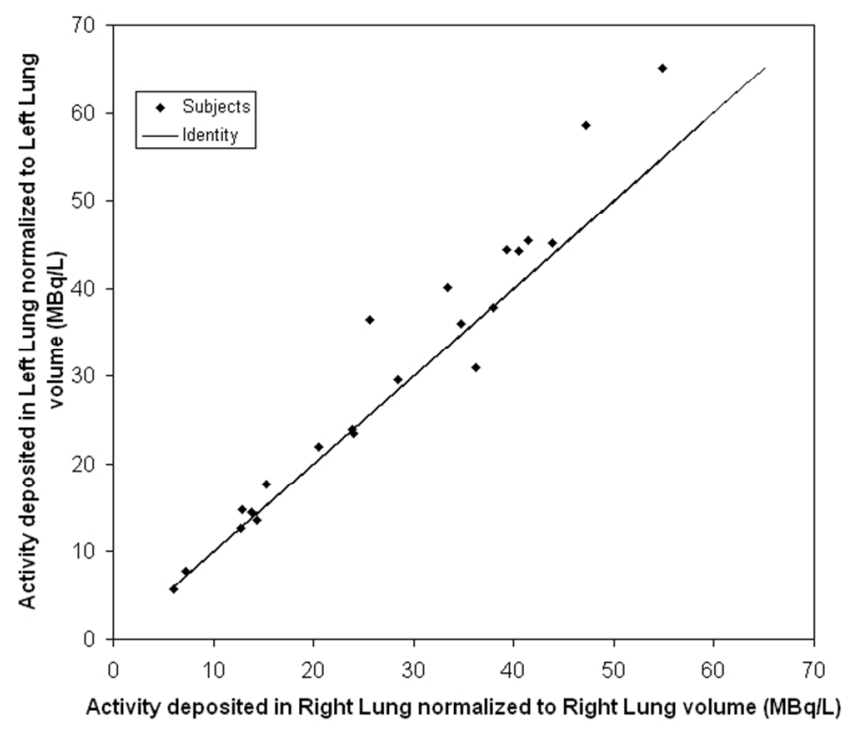

Figure 8 Deposition of activity in right and left lungs from 3D-SPECT normalized to lung volume. The line is the identity line. $256 \times 159 \mathrm{~mm}(96 \times 96$ DPI) 


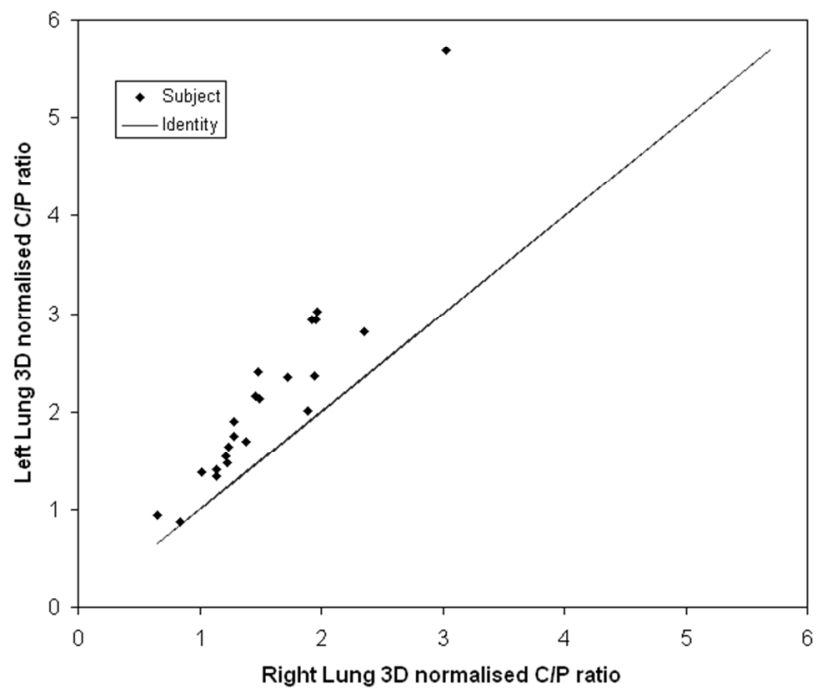

Figure 9 Spatial deposition: 3D normalized Central-to-Peripheral ratio in right and left lungs. The line is the identity line. $256 \times 159 \mathrm{~mm}(96 \times 96 \mathrm{DPI})$ 
Figure 10 Airway deposition: bronchial airways deposition fraction (BADF, generations 2-8) in right and left lungs. The line is the identity line. $256 \times 159 \mathrm{~mm}(96 \times 96 \mathrm{DPI})$ 


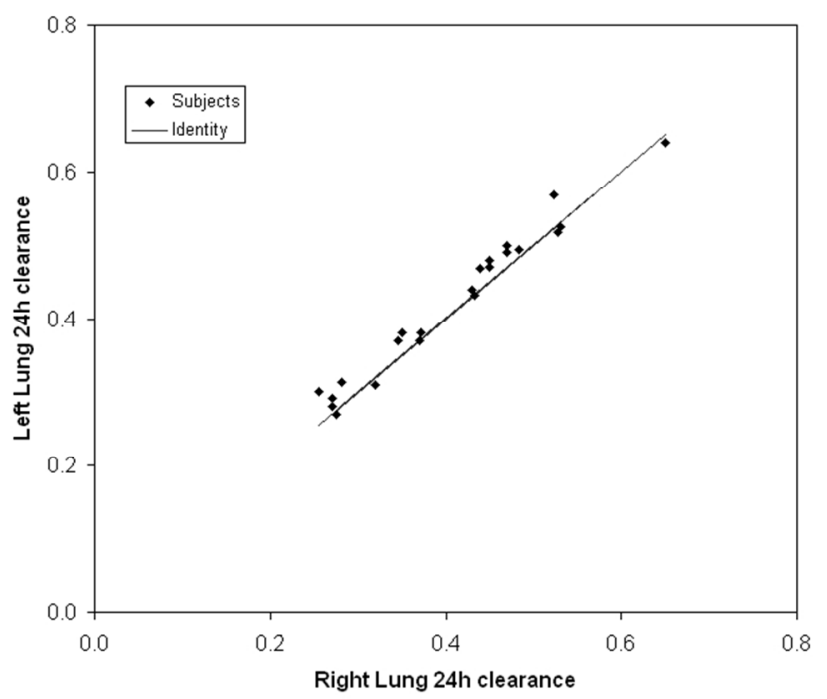

Figure $1124 \mathrm{~h}$ clearance in right and left lungs. The line is the identity line. $256 \times 159 \mathrm{~mm}(96 \times 96 \mathrm{DPI})$ 
Figure 12 The variation of $24 \mathrm{~h}$ clearance with conducting airways deposition fraction assuming the definition of the conducting airways in the Weibel Model. The parameters have been differentiated with symbols and color codes. The line is the identity line. $256 \times 159 \mathrm{~mm}(96 \times 96$ DPI $)$ 
Figure 13 Total airway deposition, ET deposition, and 24h clearance (conducting airways deposition) for large and small particles, and deep and shallow regimes. Histograms are the Means, error bars the Standard Deviation.

$173 \times 135 \mathrm{~mm}(96 \times 96 \mathrm{DPI})$ 
Figure 13 Total airway deposition, ET deposition, and 24h clearance (conducting airways deposition) for large and small particles, and deep and shallow regimes. Histograms are the Means, error bars the Standard Deviation.

$173 \times 135 \mathrm{~mm}(96 \times 96 \mathrm{DPI})$ 
Figure 13 Total airway deposition, ET deposition, and 24h clearance (conducting airways deposition) for large and small particles, and deep and shallow regimes. Histograms are the Means, error bars the Standard Deviation.

$173 \times 135 \mathrm{~mm}(96 \times 96$ DPI) 
H05A - Air

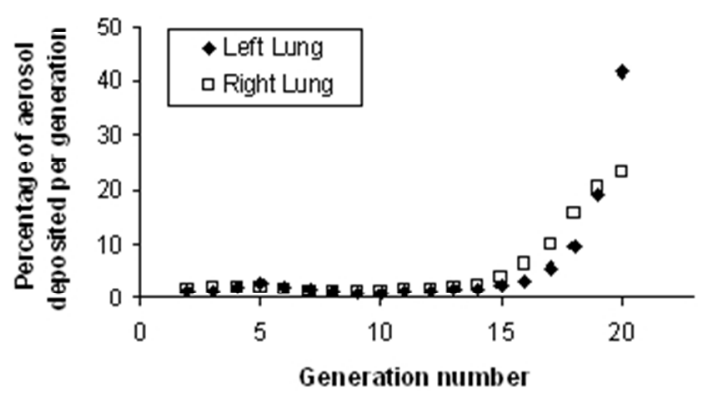

Figure 14 Generational deposition for healthy participants whose varying parameter was carrier gas. $169 \times 135 \mathrm{~mm}(96 \times 96 \mathrm{DPI})$ 


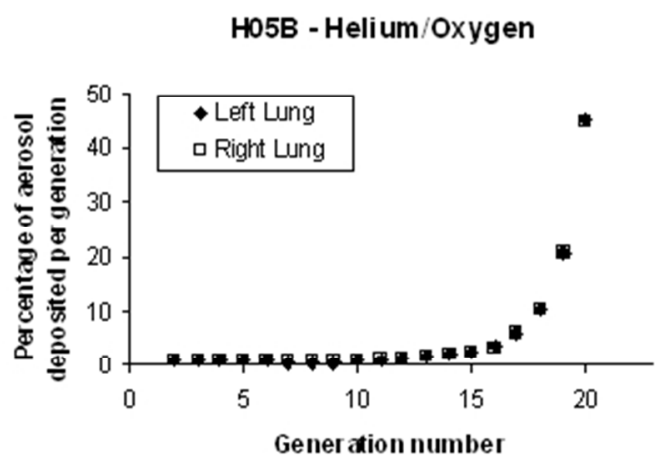

Figure 14 Generational deposition for healthy participants whose varying parameter was carrier gas. $169 \times 135 \mathrm{~mm}(96 \times 96 \mathrm{DPI})$ 
H06A - Air

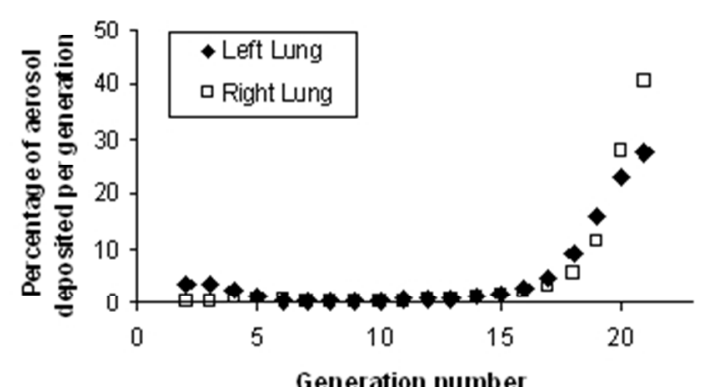

Generation number

Figure 14 Generational deposition for healthy participants whose varying parameter was carrier gas. $169 \times 135 \mathrm{~mm}(96 \times 96 \mathrm{DPI})$ 
H06B - Helium/Oxygen

Figure 14 Generational deposition for healthy participants whose varying parameter was carrier gas. $169 \times 135 \mathrm{~mm}(96 \times 96 \mathrm{DPI})$ 